\title{
Micromagnetic study of Bloch-point-mediated vortex core reversal
}

\author{
André Thiaville, ${ }^{1, *}$ José Miguel García, ${ }^{1}$ Rok Dittrich,${ }^{1,2}$ Jacques Miltat, ${ }^{1}$ and Thomas Schrefl ${ }^{2}$ \\ ${ }^{1}$ CNRS-Université Paris-sud, Laboratoire de Physique des Solides, 91405 Orsay, France \\ ${ }^{2}$ Institute of Solid State Physics, TU-Wien, Wiedner Hauptstraße 8-10, 1040 Wien, Austria
}

(Received 23 October 2002; published 18 March 2003)

\begin{abstract}
We study how micromagnetic calculations can be applied to processes that involve a singularity of the magnetization field, namely, the Bloch point. In order to allow for comparison with recent experiments, we consider Permalloy thin-film disks supporting a vortex magnetic configuration. The structure of the Bloch point at rest in the middle of the core of the vortex is studied first, comparing the evolution of the calculation results under decreasing mesh size to analytical results. The reversal of the core of the vortex under a field applied perpendicularly to the disk plane is then investigated. We apply two different procedures to evaluate switching fields and processes: direct micromagnetic time-dependent calculation, and the evaluation of the energy barrier that separates the two orientations of the vortex core in the configuration space, using a path method. Both methods show the occurrence of Bloch points during reversal. Special attention is paid to the extrapolation towards zero mesh size of the numerical results. The calculations are confronted to experimental values from Okuno et al. [J. Magn. Magn. Mater. 240, 1 (2002)]. We conclude that defects and thermal agitation are likely to assist Bloch-point injection, hence lowering the switching fields.

DOI: 10.1103/PhysRevB.67.094410

PACS number(s): 75.75.+a, 75.60.Jk, 07.05.Tp, 75.10.Hk
\end{abstract}

\section{INTRODUCTION}

In 1965, Feldtkeller first considered the consequences of the hypothesis of continuity of magnetic structures. ${ }^{1} \mathrm{He}$ showed that in certain situations, noncontinuous configurations should exist. The basic configuration of this kind is the so-called Bloch point (BP). It is defined by the following property: for any closed surface (i.e., spherelike) surrounding the point, the magnetization vectors on this surface cover the surface of the unit sphere exactly once. Topological arguments have been used to show that the BP is the only stable singularity in a ferromagnet ${ }^{2}$ when one considers all possible continuous transformations. Thus, a BP cannot appear alone within a continuous structure: either it is created in a pair, or it enters into the sample from the boundary. ${ }^{3}$

Experimental proofs of the existence of BP's are not numerous, but exist. Lorentz imaging of nickel thin films with an asymmetric Bloch-wall structure showed polarity reversals that imply the presence of a BP. ${ }^{4}$ Mobility data of bubble domains in garnet films could only be explained with BPcontaining structures. ${ }^{5}$ The observation of Bloch line vibration in yttrium iron garnet (YIG) crystals showed directly the injection of a BP's into the line. ${ }^{6}$ Observation of Bloch line displacement under pulsed fields in bubble garnet films allowed to monitor the BP injection and the presence of BPs in such lines. ${ }^{7}$ Therefore, no doubt exists about the real existence of the Bloch point. We anticipate that, as the lateral dimensions of the magnetic samples prepared using nanofabrication techniques shrink towards the characteristic length of micromagnetics, the impact of the presence of one Bloch point in the sample will dramatically increase. Thus, micromagnetic studies of processes that involve a Bloch point should be considered.

The paper's layout is as follows. The introduction goes on recalling the results of previous work, analytic or numeric, about the BP structure and energy. Section II is devoted to numerical calculations of the BP at rest within a vortex mag- netic structure, the sample being a Permalloy circular disk. The following section shows that a BP is involved in the switching of the magnetization of the vortex core, under a field opposing the core magnetization. Finally, as the BP is a singular structure, the mesh size dependence of the results obtained by numerical calculations at a finite mesh is studied in detail.

The modeling of this singular structure is not yet fully satisfactory. The definition of the BP implies that the modulus of the magnetization must vanish at the BP center, so that micromagnetics does not apply in this region. Indeed, micromagnetics is derived from atomic models under the assumption of a continuous magnetization distribution, varying only slowly on the atomic scale. ${ }^{8}$ Considering a sphere surrounding the BP and sufficiently far from it so as to apply micromagnetic theory, Feldtkeller ${ }^{1}$ showed that the leading energy density is exchange energy. This term is smallest when the magnetization direction at a given point is the unit vector from the BP to the point considered. A rotation of all moments by the same amount does not change the exchange energy. In that case, the exchange energy density amounts to

$$
d E_{A}=\left(2 A / r^{2}\right) r^{2} d r \sin \theta d \theta d \varphi,
$$

with $r, \theta$, and $\varphi$ being the spherical coordinates for the position and $A$ the exchange constant. It is immediate to note that, although the energy density diverges at the origin, the integrated exchange energy within a sphere of radius $R$ is finite,

$$
E_{A}(R)=8 \pi A R .
$$

Döring then showed ${ }^{9}$ that the next energy term is the demagnetizing energy. It can be minimized for a nonzero rotation angle of magnetization vectors with respect to the local position unit vector (Fig. 1). On these bases, Slonczewski and co-worker elaborated the first approximate calculation of the BP energy in the case of a BP inside a domain wall in a 


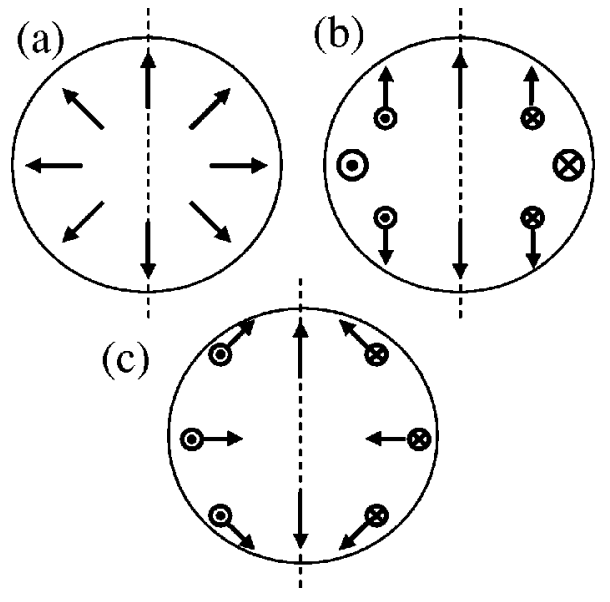

FIG. 1. Three schematic configurations for a Bloch point, having a revolution axis (vertical axis of the figure). (a) is the hedgehog $\mathrm{BP}$, (b) the circulating BP, and (c) could be called the spiraling BP. The configuration (a) has the highest magnetostatic energy, as it builds up a large monopole in the center. This monopole charge is reduced in (b) by a $90^{\circ}$ rotation of the moments around the axis. The spiraling of the magnetization in (c), obtained by a rotation of angle larger than $90^{\circ}$, provides opposite charges in the core surrounding that decrease further the magnetostatic energy.

bubble garnet film. ${ }^{10,11}$ The result, in the limit $Q$ $=2 K_{u} / \mu_{0} M_{s}^{2} \gg 1\left(K_{u}\right.$ is the uniaxial anisotropy constant, the easy axis being perpendicular to the film plane), reads

$$
E_{B P}=2 \pi A \sqrt{A / K_{u}}(1.90+\ln Q) .
$$

The logarithm of the quality factor $Q$ expresses the fact that, for a smaller magnetization, the BP would spread within the domain-wall plane. Comparing to Eq. (2), one sees that for a typical bubble garnet quality factor $Q=10$, the BP radius $R$ is of the order of $1 / 3$ of the domain-wall width $\pi \sqrt{A / K_{u}}$. A similar result was obtained by Hubert, using a Ritz method to evaluate approximate energies and structures. ${ }^{12}$

If we turn now to the structure and energy of the core of a BP, where the magnetization falls to zero, only crude estimates exist. Assume, for example, that the central atom becomes nonmagnetic. In a simple cubic(sc) lattice, with $J$ being the Heisenberg exchange energy and $a_{0}$ the lattice constant, the energy cost is linked to the number of broken bonds (six here): $\Delta E_{\text {core }}=6 \mathrm{~J}$. On such a lattice, the familiar transformation to continuous structures relates $J$ to the exchange constant $A$ by $A=J / 2 a_{0}$, so that one estimates the core energy as

$$
\Delta E_{\text {core }}=12 A a_{0} \approx 8 \pi A\left(a_{0} / 2\right) .
$$

Comparing to Eq. (2), this correction is small as soon as the radius $R$ is much larger than the lattice constant $a_{0}$, which is often the case, as discussed later. Note that the same type of calculation, with a nonmagnetic region of variable size, shows that this region is of the order of one atom in size, justifying the above evaluation.

A related calculation was performed by Reinhardt who computed classically the exchange energy around the BP, using a Heisenberg formulation ${ }^{13}$ (such a formulation does not rely on the assumptions of a continuous and slowly varying magnetization distribution). Comparing to the result of the continuous calculation, he found that the atomiclike formulation gave a lower energy, the variation reading

$$
\Delta E_{\text {discrete }}=-A a_{0} \Delta\left(\vec{r}_{0}\right),
$$

with the function $\Delta$ depending slightly on the exact location $\vec{r}_{0}$ of the BP within the lattice. As he computed $\Delta \approx 13$ for a sc lattice, the correction is nearly equal and opposite to the core energy given by Eq. (4). Therefore, as already stated by Hubert, ${ }^{12}$ the core energy corrections can be estimated to be much smaller than the micromagnetic contribution, as soon as the extent of the BP micromagnetic structure is large compared to atomic distances. This justifies undertaking micromagnetic calculations with Bloch points. Note finally that first-principles calculations with noncollinear moments should in the future become able to compute BP cores.

Full micromagnetic calculations that contain BP's are very rare; apparently only Nakatani and Hayashi published papers on this subject. In one case, the static structure of a bubble garnet sample containing one wall, one line, and one BP was calculated. ${ }^{14}$ This is the same geometry considered by Slonczewski and co-worker ${ }^{10,11}$ and Hubert. ${ }^{12}$ The BP was compelled to sit in the center, with $3 \times 3 \times 3$ cells having a given magnetization corresponding to the circulating BP structure and with no moment in the center. However, apart from looking at the resultant structure, no analysis of the results was performed regarding energies, comparison to analytic results, and mesh size dependence. The second paper $^{15}$ considered the dynamics of the same kind of structures, but with an approximate demagnetizing field calculation which is appropriate for a domain-wall geometry. No mesh cells had fixed magnetization vectors, and it was observed that a BP could enter at one film surface and propagate between the mesh points. These two calculations have demonstrated that micromagnetic calculations involving BP's are technically feasible, but did not give an appreciation of their physical validity.

This last point is the subject of the paper. We consider a different geometry, namely, a disk-shaped Permalloy sample, of such size so as to develop a vortex structure. In a first part, we investigate the micromagnetic structure of the BP at rest within such a structure (in fact, in an unstable equilibrium). The mesh size $d$ is systematically varied, always below the exchange length $\Lambda$ (defined as $\sqrt{2 A / \mu_{0} M_{s}^{2}}$ ), and the results are compared to the analytical formulas given above. The second part deals with the reversal of the vortex core under a field applied perpendicularly to the disk plane. This situation corresponds to recent experiments performed by Okuno et $a l .,{ }^{16}$ in which the vortex core orientation is observed by MFM (magnetic force microscopy) in the remanent state. An array of Permalloy disks is submitted to an applied field for a macroscopic duration (1 min typically), and a subsequent image allows to count the number of cores that have switched. A switching field distribution is thus measured, for a series of disk diameters at a fixed thickness of $50 \mathrm{~nm},{ }^{16}$ and also more recently for a varying film thickness. ${ }^{17}$ 
TABLE I. Total energy of a Bloch point for disks of varying diameter, $50 \mathrm{~nm}$ thickness, and for various mesh sizes. These BP insertion energies are all evaluated as the excess of the corresponding energies for a vortex with a BP in the center over that of a vortex with no BP. The parameter $b$ in Eq. (6) that allowed to equilibrate the BP structure is also indicated.

\begin{tabular}{cccccc}
\hline \hline $\begin{array}{c}\text { Diameter } \\
(\mathrm{nm})\end{array}$ & $\begin{array}{c}\text { Mesh } \\
\left(\mathrm{nm}^{3}\right)\end{array}$ & $\begin{array}{c}E_{\text {tot }} \\
\left(10^{-18} \mathrm{~J}\right)\end{array}$ & $\begin{array}{c}E_{\text {exc }} \\
\left(10^{-18} \mathrm{~J}\right)\end{array}$ & $\begin{array}{c}E_{\text {dem }} \\
\left(10^{-18} \mathrm{~J}\right)\end{array}$ & $\begin{array}{c}b^{2} \\
\left(\mathrm{~nm}^{2}\right)\end{array}$ \\
\hline 100 & $2.5^{3}$ & 1.86 & 1.98 & -0.12 & 93. \\
& $3.125^{3}$ & 1.78 & 1.90 & -0.12 & 95.406 \\
104 & $4 \times 4 \times 5$ & 1.66 & 1.79 & -0.13 & 83.052 \\
200 & $2.5^{3}$ & 1.93 & 2.07 & -0.14 & 93. \\
& $3.125^{3}$ & 1.84 & 1.99 & -0.15 & 92.952 \\
& $4 \times 4 \times 5$ & 1.70 & 1.85 & -0.15 & 81.395 \\
400 & $4 \times 4 \times 5$ & 1.70 & 1.85 & -0.15 & 81.465 \\
\hline \hline
\end{tabular}

\section{MICROMAGNETIC CALCULATION OF A BP AT REST}

For bubble garnet films, it has been shown that for large film thicknesses compared to the exchange length, the line containing a BP has lower energy than a line without BP, for magnetostatic reasons. ${ }^{12}$ This is not true in the case of a vortex in a disk, at least for the sizes of the samples of Okuno et al. Nevertheless, we have found that it is possible to stabilize a vortex core that contains a BP exactly in its center. A starting configuration was generated by an analytical guess, reading

$$
\begin{aligned}
& m_{x}=-\frac{y}{\sqrt{x^{2}+y^{2}}} \sqrt{1-m_{z}^{2}}, \\
& m_{y}=\frac{x}{\sqrt{x^{2}+y^{2}}} \sqrt{1-m_{z}^{2}}, \\
& m_{z}=\frac{z}{r} \frac{b^{2}}{b^{2}+x^{2}+y^{2}} .
\end{aligned}
$$

In what follows, $z$ denotes the normal to the disk plane. The adjustable parameter $b$ corresponds to the size of the BP structure. For an in-plane distance well below $b$, one recovers the analytic structure of a circulating BP, whereas well above $b$ the magnetization lies in the plane and is circular. To successfully converge to the BP structure, the parameter $b$ has to be optimized for each case (disk diameter, thickness, mesh size): values too large or too small lead to BP expulsion. For the calculations presented below, this parameter was close to $10 \mathrm{~nm}$. Depending on the number of mesh cells across the thickness, the parameter $b$ had to be optimized with more or less precision (see Table I). In fact, successful convergence into this unstable equilibrium configuration was possible because of the existence of a "mesh friction." It can be directly understood from the Reinhardt calculation results, in which the atomic lattice is replaced by the numerical mesh. These calculations show that the BP prefers to sit farthest from mesh points: in the sc lattice $\Delta\left(\vec{r}_{0}\right)$ is minimum at 13.4 (at the body center), and has a saddle value equal to
13.0 at the center of a facet. ${ }^{13}$ The resulting mesh-friction barrier is thus $0.4 A d$ (where $d$ is the edge size of the mesh cell), which results in a numerical value of $1 k_{B} T_{a m b}$ per nanometer for $d$. This creates a tiny potential well at every place in between the mesh points. For these calculations, it was also necessary to have an even number of cells in all directions, so that the BP could be placed exactly in the center of the sample.

The numerical calculations were performed with the public micromagnetic program OOMMF. ${ }^{18}$ Mesh cells were cubic ( $d=2,2.5$, and $3.125 \mathrm{~nm}$ ), except for the larger one that was tetragonal (cell size $4 \times 4 \times 5 \mathrm{~nm}^{3}$ ). Exchange interaction is computed using the six nearest neighbors and in an Heisenberg-like fashion. This formulation has the advantage of never diverging whatever the structure is. ${ }^{19}$ Moreover, it allows a direct comparison to atomiclike calculations as discussed above. The demagnetizing field is evaluated under the assumption of a constant magnetization within each cell. Material constants representative of Permalloy were adopted, namely, exchange constant $A=10^{-11} \mathrm{~J} / \mathrm{m}$, magnetization $M_{s}=800 \times 10^{3} \mathrm{~A} / \mathrm{m}$, zero anisotropy, and gyromagnetic ratio $\gamma_{0}=2.21 \times 10^{5} \mathrm{~m} \mathrm{~A}^{-1} \mathrm{~s}^{-1}$ (we use SI units throughout). The damping constant was in most cases taken as $\alpha=0.5$ (suitable for quasistatic calculations where one looks for the equilibrium), but for some dynamic calculations a more realistic value $\alpha=0.01$ was used. The convergence criterion for quasistatic calculations was that $|d \vec{m} / d t|<0.01 \%$ ns at every mesh point. From the Landau-Lifchitz-Gilbert equation, this is equivalent to $\left|\vec{H}_{e f f} \times \vec{m}\right| / M_{s}<1.1 \times 10^{-6}$.

First we consider total energies, without looking at their distribution in space. Table I shows the calculated values for disks $50 \mathrm{~nm}$ thick. The BP energy is evaluated by difference, comparing a vortex with a BP to a vortex without BP. Total energies are about $1.8 \times 10^{-18} \mathrm{~J}$. This is more than $400 k_{B} T$ (at $300 \mathrm{~K}$ one has $k_{B} T=4.14 \times 10^{-21} \mathrm{~J}$ ), so that pairs of BP's will never appear spontaneously. The exchange energy is the dominant contribution, the magnetostatic energy being about only $8 \%$ of the total. Note that this last contribution is negative, whereas intuition based on the interaction of two bar magnets (one for each half of the vortex core) would predict a positive energy. However, the structure of the vortex is much more complex than this simple picture: ${ }^{20}$ a partial flux closure develops around the core, and the core width is affected by the exterior (for example, the core width depends on the film thickness, and on the position across the thickness). Therefore, the more appropriate intuitive image would be that of two bar magnets with some soft material surrounding them. The reader can verify by direct experiment that, whereas two opposite magnets repel, placing a large enough soft sphere in between results in an attraction at short distance. ${ }^{21}$ The relative importance of these two contributions is also in agreement with the previous work recalled in Sec. I.

The calculations show some dependence on mesh size. In particular, the exchange energy increases almost linearly with decreasing mesh size. In order to understand the reasons for this increase, we turn to a local analysis of the exchange energy. For this, we consider spheres centered on the BP and 


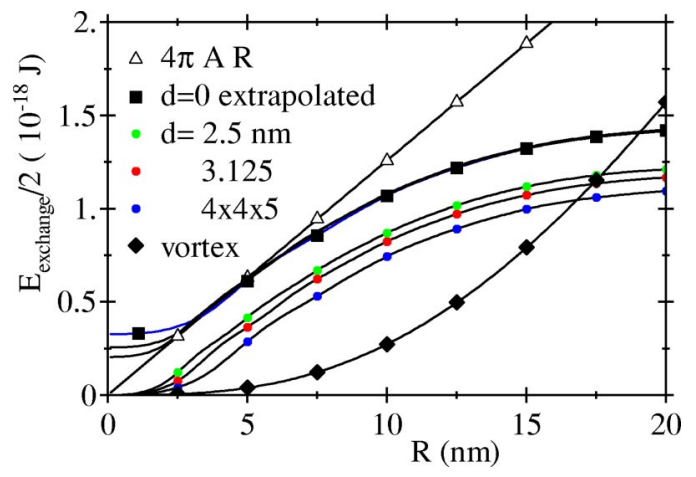

FIG. 2. Local exchange energy of a Bloch point. The exchange energy distribution is evaluated within spheres of increasing radius $R$, located at the film center (where the $\mathrm{BP}$ is). BP exchange energy is evaluated as the energy difference between a vortex having a BP and one without it, for a series of mesh sizes $d$. The profiles are compared to the analytical relation (2), and a linear extrapolation to zero mesh size of these profiles is shown (see formula in the text; the symbol was put on the extrapolation from the coarsest mesh). The profile for the vortex without BP is also shown (at $d$ $=2.5 \mathrm{~nm}$ ). The sample is a 200-nm-diameter disk, $50 \mathrm{~nm}$ thick. The vertical axis shows the half-energy for later comparison with the corresponding profiles at a surface of a disk containing a compressed vortex.

of increasing radius. All calculation cells are scanned and their volume contained within the sphere evaluated. The exchange energy embedded in the sphere is then obtained as the sum over all cells of their local exchange energy density times the contained volume. The local exchange energy density is given by the Heisenberg term summed over all next nearest neighbors (as a check, we also recalculated the exchange energy using a five point polynomial interpolation method in each direction, ${ }^{22}$ and obtained a value of BP exchange energy lower by only $2 \times 10^{-20} \mathrm{~J}$ ). Figure 2 is a plot of this local exchange energy versus the radius of the sphere, for a disk diameter of $200 \mathrm{~nm}$ and a thickness of $50 \mathrm{~nm}$. All curves show three regions. For a radius below one mesh size, the exchange energy rises very slowly (as the volume of the sphere). This is a consequence of the nonzero mesh parameter, and of the way we chose to evaluate local exchange energy. In an intermediate regime, the exchange energy rises linearly. The slope is that of the analytic relation (2), with a lateral offset that proves equal to $0.65 d$. Finally the energy saturates at a larger radius, meaning that the BP embedded in a vortex has a given size. The typical radius of the BP structure is $10 \mathrm{~nm}$, in agreement with the parameter $b$ found when using the analytical structure (6). For a radius larger than 20 $\mathrm{nm}$ (at this thickness), the BP exchange energy decreases a little so as to reach the total energy values shown in Table I. In this regime the sphere is partly out of the sample, and one also probes the flux closure region far away from the core. Thus, the local evaluation of the exchange energy is probably more adapted to the study of the mechanism of BP injection.

Figure 2 also displays a linear extrapolation of the exchange energy profiles to zero mesh size, obtained by adding an energy $4 \pi A(0.65 d)$ to the three computed profiles (with $d=4 \mathrm{~nm}$ in the larger mesh case). The extrapolated profiles merge tangentially into the analytical one at about $5 \mathrm{~nm}$, and are above it for low $R$ because a constant was added. These plots show directly that, roughly speaking, the numerical calculations miss the part of the exchange energy that corresponds to a radius $R<0.65 d$. Moreover, this proves that the analytic theory describes well the vicinity of a Bloch point. Last, as the typical BP radius is $10 \mathrm{~nm}$, it is much larger than the lattice constant so that the core energy corrections are anticipated to play a minor role.

Figure 3 compares the structures of a vortex without and with a BP, in the form of a series of slices across the sample thickness. The vortex core radius varies across the thickness according to the so-called barrel shape already found by approximate Ritz calculations. ${ }^{20}$ Moreover, close to the surfaces the in-plane magnetization flow adopts the form of spirals so as to screen the magnetic surface charges associated with the core magnetization (see also Fig. 4). The spiraling is markedly reduced for a structure with a $\mathrm{BP}$, because the $\mathrm{BP}$ itself compensates the surface charges (Fig. 4). This is re-
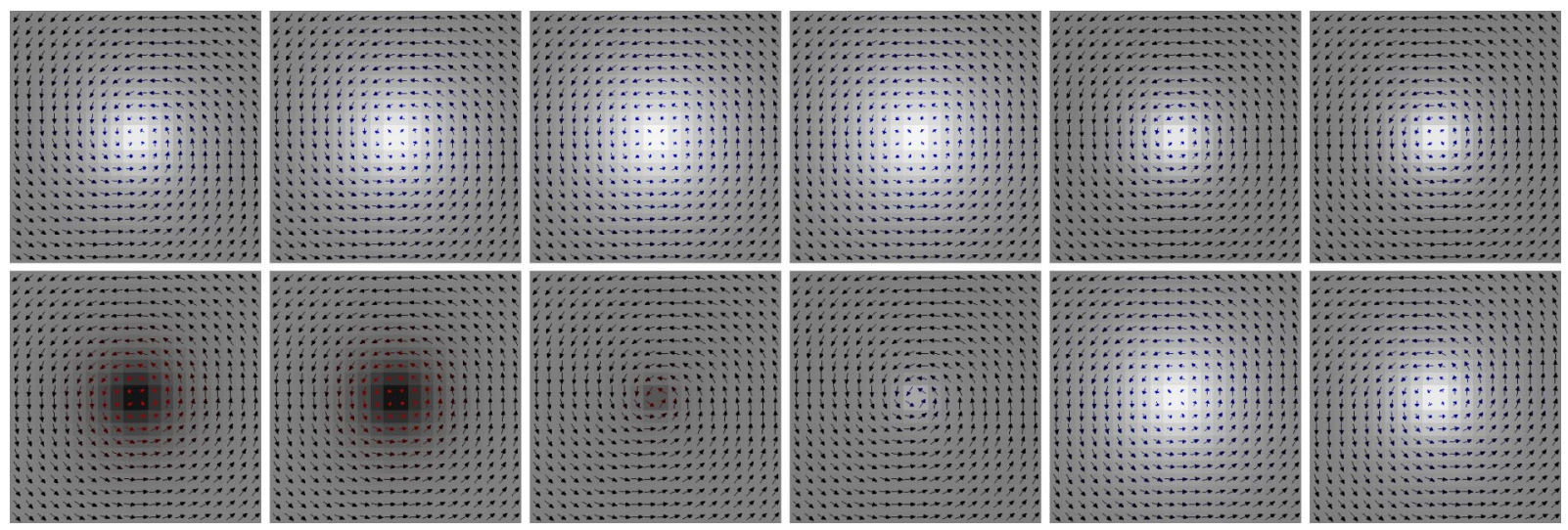

FIG. 3. Views of the central part (50 nm squares) of the structure of a normal vortex (top series) and of a vortex with a Bloch point (bottom series), in the form of a series of slices at different depths. The sample is $200 \mathrm{~nm}$ in diameter, $50 \mathrm{~nm}$ thick, and with a 2.5 -nm mesh. Slices pertain, from left to right, to the cell planes number 1 (bottom), 5, 10, 11, 15, and 20 (top). Pixels are colored according to the perpendicular magnetization component (the pure vortex core is upwards magnetized), while the in-plane magnetization orientation is given by the arrows. The BP structure is of type (c) shown in Fig. 1. 


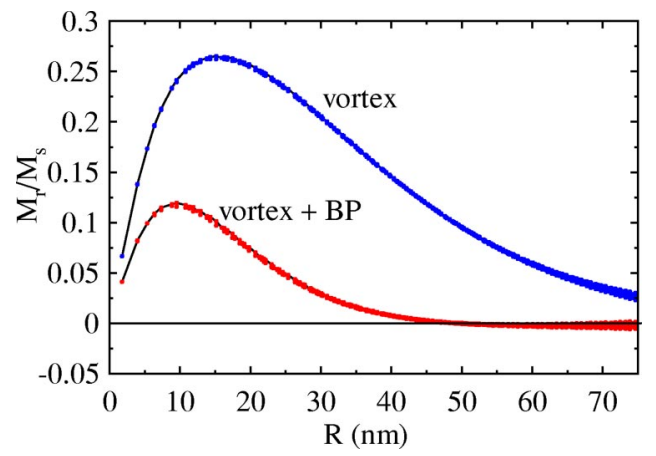

FIG. 4. Plot of the radial magnetization component at the surface of a disk of diameter $200 \mathrm{~nm}$ and thickness $50 \mathrm{~nm}$, with a mesh size of $2.5 \mathrm{~nm}$. The values are radially averaged. The pure vortex shows a relatively large radial deviation of the magnetization, which has an opposite sign at the other surface (not shown). This deviation is much smaller when a BP is present, because of partial charge compensation.

lated to the lower magnetostatic energy of a vortex having a BP compared to a pure vortex.

\section{THE SWITCHING OF VORTEX CORES}

\section{A. Quasistatic calculations}

The switching of the magnetization of the vortex core under a perpendicular field was investigated by quasistatic calculations: the field was increased by steps ( $4 \mathrm{mT}$ typically) and equilibrium reached at each step. The field was applied at a small angle $\left(1^{\circ}\right)$ from the disk normal so as to avoid symmetry-related artifacts. Experiments ${ }^{16}$ have shown that only for large angles, typically greater than $15^{\circ}$, the vortex can be expelled before its core switches. Furthermore, a value of $1^{\circ}$ is representative of the accuracy of the orientation of the field in the experiments.

Figure 5 shows the computed magnetization curves for a 200-nm-diameter disk, of $50 \mathrm{~nm}$ thickness. A quasilinear slope is seen, followed by saturation. For the sake of completeness, results of two-dimensional (2D) computations (prismatic cells of height $50 \mathrm{~nm}$ and edge sizes of 2 or $4 \mathrm{~nm}$ ) are also given. Worth noting is the difference in the saturation region: the 2D calculations saturate to $M_{s}$, while a lower value is found in $3 \mathrm{D}$ in the same field region. This corresponds to the magnetization fanning at the disk edges into a flower state. The apparent saturation reached in $3 \mathrm{D}$ under an induction of $0.6 \mathrm{~T}$ is not full, and the flower state goes slowly to a uniform state as the field rises. The 2D calculations, with only one cell across the thickness, cannot describe the flower state, so that they reach full saturation at a lower field compared to the 3D calculations. They can neither describe processes that occur inhomogeneously across the thickness of the sample, so that from now on we shall only consider results of 3D calculations.

On these curves, vortex core reversal is seen as a tiny step, since the relative volume of the core is small at this sample size (inset, Fig. 5). The computed reversal fields change very rapidly with mesh size for the 2D calculations, whereas the evolution is not as fast for the $3 \mathrm{D}$ results. Pic-

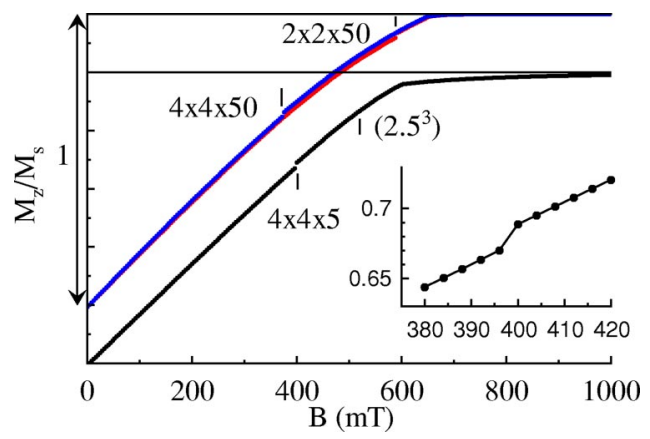

FIG. 5. Computed magnetization curves for a vortex in a 50nm-thick disk, $200 \mathrm{~nm}$ in diameter, with a negative core magnetization. The field is applied perpendicularly in the opposite direction, at an angle of $1^{\circ}$ from the axis, in steps of $4 \mathrm{mT}$. The computations were performed in $2 \mathrm{D}$ and $3 \mathrm{D}$, for different meshes (curves were offset vertically by 0.2 for clarity). The core reversal appears as a small step in the otherwise rather linear curve (see inset corresponding to the $3 \mathrm{D}$ case, where the points are the calculated results). Mesh refinement changes only the core reversal field, but by a large amount. For the $2.5-\mathrm{nm}$ mesh in $3 \mathrm{D}$, only the core reversal field is indicated. The low value of the apparent saturation in the 3D curve (at about $0.6 \mathrm{~T}$ ) is ascribed to the presence of a flower state.

tures of the magnetization distribution at different fields are shown in Fig. 6. The remagnetization of the structure is seen to proceed from the sample edges. The core moves slightly under the action of the in-plane component of the field, and is more and more compressed by the perpendicular component. During this process the exchange energy increases. This excess of exchange energy will drive later the insertion of a BP. The vortex structure remains after the core has switched, up to a quasisaturation close to $600 \mathrm{mT}$ : it is still magnetostatically favorable.

Figure 7 is a summary of the calculated core switching fields under these conditions (field at $1^{\circ}$ off the normal, thickness $50 \mathrm{~nm}$ ) versus mesh size and for several diameters. The general tendency is that the calculated switching field increases as the mesh size is reduced. The large switching fields for higher aspect ratio (diameter/thickness) samples can be qualitatively understood as an internal field effect: a flatter sample has a larger demagnetizing factor in the perpendicular direction, hence a lower internal field (although, as the structure is very nonuniform at the vortex core, the argument is simplistic).

The process by which reversal occurs is now investigated in detail, and we will show that it involves one BP. Figure 8 corresponds to the reversal of a vortex core in a 100-nmdiameter 50-nm-thick disk, the mesh size being $2.5 \mathrm{~nm}$. Under a field of $331 \mathrm{mT}$ applied at $1^{\circ}$ from the disk normal, the core was not yet reversed. In the following we are interested in the time evolution of the magnetization and exchange energy during the reversal of the vortex core. The time is set to zero and the field is increased from $331 \mathrm{mT}$ to $332 \mathrm{mT}$. At this field the switching of the core occurs. The calculation is run with the same damping constant as before, namely, $\alpha=0.5$. The perpendicular magnetization is seen to decrease rapidly, between $t=1360$ and $t=1420 \mathrm{ps}$ [Fig. 8(a)]. The decrease, averaged over the sample volume, amounts to 


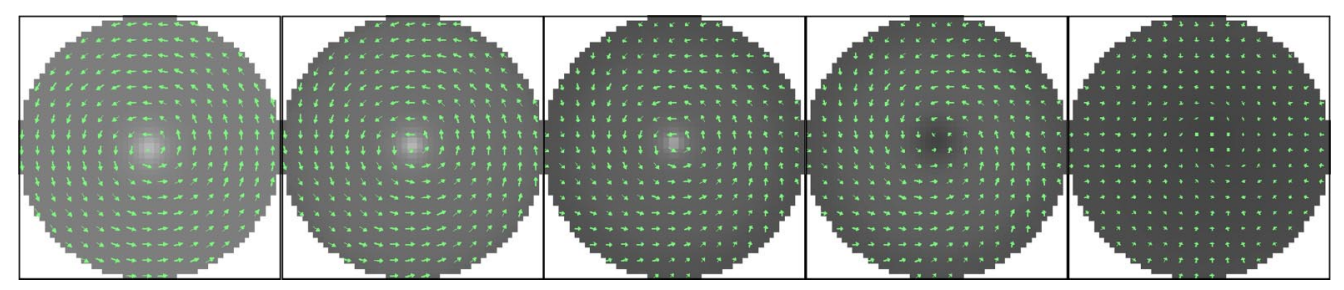

FIG. 6. Pictures of the magnetic structure in a 200-nm disk starting from the vortex state in zero field and subjected to a perpendicular field ( $1^{\circ}$ off in the horizontal direction) opposite to the core magnetization (thickness $50 \mathrm{~nm}$, mesh size $4 \times 4 \times 5 \mathrm{~nm}^{3}$, field step $4 \mathrm{mT}$ ). The fields are, from left to right, 0, 200, 396 (just before the core reversal), 400 (just after core reversal), and $600 \mathrm{mT}$ (just after the vortex structure has disappeared). The views correspond to the top plane of the sample, and the magnetization is displayed like in Fig. 3.

$5 \times 10^{4} \mathrm{~A} / \mathrm{m}$. This value is close to twice that of the vortex in zero field $\left(3.4 \times 10^{4} \mathrm{~A} / \mathrm{m}\right)$, meaning that the compressed vortex core completely reverses. In the same figure, the plot of the total exchange energy shows a faster decrease, between $t=1360$ and $t=1380 \mathrm{ps}$. The step in the exchange energy amounts to $10 \times 10^{-18} \mathrm{~J}$, five times as large as the exchange energy in the BP for this sample (Table I). Thus, there is enough energy stored in the compressed vortex to afford BP injection. Moreover, a strong energy barrier has to exist so as to prevent this injection from occurring at much lower fields. As a simple calculation shows, the ratio of 5 means that the average exchange energy density in the compressed vortex core is close to that in the BP structure.

Figure 8(b) examines this fast reversal process more closely. The maximum of magnetization velocity (proportional to maximum torque), $\max (|d \vec{m} / d t|)$, shows high values in the time interval $t=1360$ to $t=1380 \mathrm{ps}$. During this period, direct inspection of the magnetization transient configurations shows a BP entering the sample at the top surface, and traveling across the thickness (Fig. 9).

The torque curve also shows clear and regular oscilllations. Correlation with the transient magnetization images (Fig. 9) showed that they correspond to a BP moving across the various cell planes. This shows the effect of mesh friction mentioned earlier. A post-treatment of the magnetization files was performed in order to detect the presence of BP's more quantitatively (see the Appendix). The resulting BP position

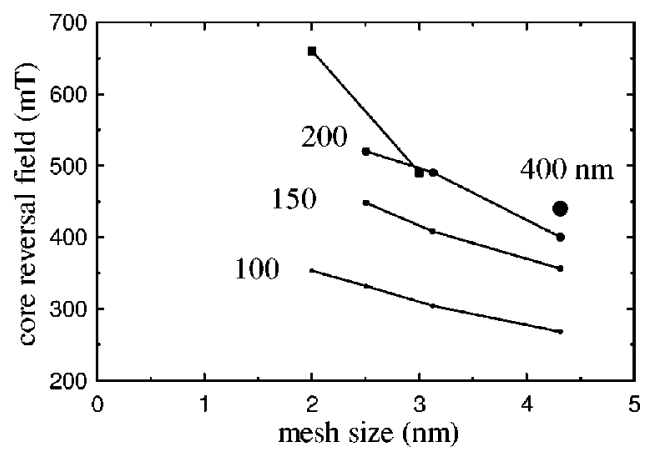

FIG. 7. Plot of the calculated core reversal fields for 50-nmthick disks, and diameters 400, 200, 150 and $100 \mathrm{~nm}$ (symbol size shrinks accordingly), versus the mesh size used in the 3D calculations. The fields always increase upon mesh refinement. All points were derived by quasistatic calculations, except for the two square symbols that denote extrapolated zero barriers at the diameter of $200 \mathrm{~nm}$ (see Sec. III B). is also plotted in Fig. 8(b). It shows one BP appearing at the top surface and crossing the whole thickness of the film. The arch tops on the torque curve correspond to the BP crossing successive planes of mesh points. The final big peak corresponds to the moment where the BP leaves the sample, at the bottom surface. The reversal has also been computed with a more reasonable value of the damping constant $(\alpha=0.01)$. The features are very similar, with a sole increase of the delay before core reversal.

Figure 10 shows the case of a disk of $200 \mathrm{~nm}$ diameter, with similar thickness and mesh, for a damping constant $\alpha=0.5$. The BP localization procedure indicates that a first BP enters from the top surface at $t=550 \mathrm{ps}$, and a second one from the bottom surface at $565 \mathrm{ps}$, both annihilating at $t=575 \mathrm{ps}$. The displacement of the BP's through the lattice gives rise to torque oscillations, and a large peak signals their annihilation.

In the dynamic simulations performed for a variety of disk diameters, thickness, and mesh sizes, the successive appearance of two BP's was most often seen. There is indeed no reason, in a quasistatic calculation where precession effects are negligible, to break the equivalence of the two film surfaces. On the other hand, energy conservation would favor the injection of a single point (see Sec. III B). Moreover in dynamics, precession magnifies the small asymmetry between the top and bottom surfaces, which originally arises from the screening of surface charges (Figs. 4 and 11). As a result, the first BP always enters through the surface where the moments acquire larger radial components by precession. In one case, finally, a pair of BP's was created in the middle of the film (diameter $100 \mathrm{~nm}$, thickness $50 \mathrm{~nm}$, mesh of 2 $\mathrm{nm}$, starting field $353 \mathrm{mT}$, applied field $353.5 \mathrm{mT}, \alpha=0.5$ ).

Let us now try to understand the variation of the core switching field with mesh size. It is appropriate here to quote Aharoni. ${ }^{23}$ "It is impossible to keep the computer from converging into the 'wrong' minimum, at all stages of the computation. In particular, when it becomes necessary to 'jump' from one branch of the energy manifold to another, there is very little indication on how to guide the computer on where to look for such a jump, and to which branch to move. It is a mistake to allow the computer to make all the decisions, and it cannot lead to a reliable result which has a physical meaning." Thus, it is important to have a check of the calculations so as to know whether the computed switching fields have a physical meaning or not. This question is especially important when one deals with singular structures such as the BP. 

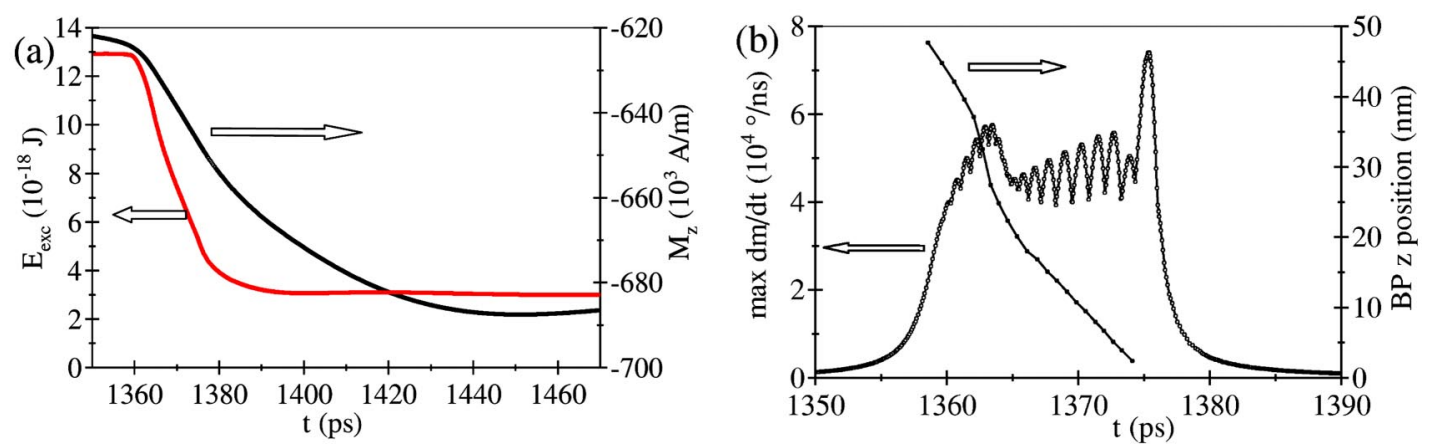

FIG. 8. The dynamics of vortex core reversal in a 50-nm-thick disk with a diameter of $100 \mathrm{~nm}$, in a high damping case $(\alpha=0.5)$, mesh of $2.5 \mathrm{~nm}$. The structure was equilibrated step by step up to a perpendicular field of $331 \mathrm{mT}$ (at $1^{\circ}$ from the normal), and the field was raised to $332 \mathrm{mT}$ at $t=0$. The reversal is seen both on the magnetization and the exchange energy (a). The BP localization program (see the Appendix) finds a single BP crossing all the film; a strong torque is present at this moment which shows some oscillations as the BP crosses the cell planes (b). Note the dilated time scale in (b).

We propose to analyze the field evolution of the structures by computing local exchange energies, comparing them to the local exchange energy of a BP calculated above. As an isolated BP has to enter the film at one surface, we choose to evaluate the local exchange energy within half spheres stuck to the sample top surface. In the following section we will show that the state at the barrier top for the BP introduction is indeed that with a BP at the surface, when the applied field reaches the switching field. The sphere center is chosen to be the average geometric center of the vortex, for the range of fields considered. This center is determined from the $z$-averaged $x y$ components of the magnetization (it differs from the center of the disk because the field is not applied perpendicularly). Figure 12 shows the evolution of the local exchange energy profiles with the applied field. The antiparallel field (counted positive) induces a compression of the

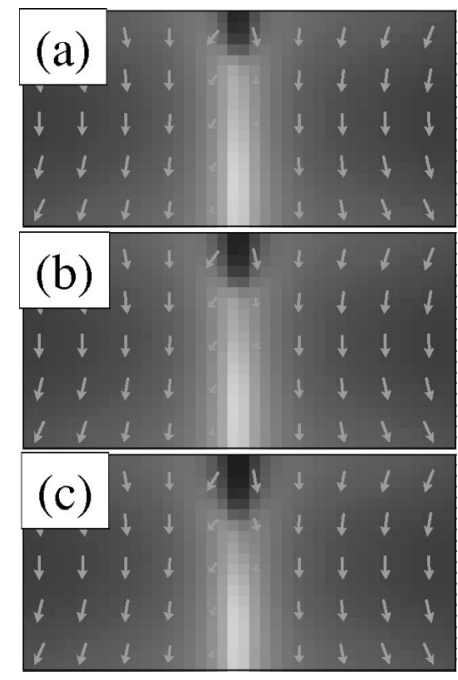

FIG. 9. Transient images (cross section) showing the displacement of a BP during the vortex core reversal in a Permalloy disk, corresponding to Fig. 8. The images were taken at times $t$ $=1362.0 \mathrm{ps}$ (a), $1362.7 \mathrm{ps}$ (b) and $1363.3 \mathrm{ps}$ (c). The gray scale reflects the magnetization $z$ component. The plane of the cross section is the 21 st cell plane, which means just above the center of the disk. vortex core, seen as a displacement of these profiles towards larger values. The profiles are close to a $R^{2}$ law, with a prefactor that increases faster than linearly with field. The profiles for one parallel field (counted negative) are also drawn in Fig. 12. As such a field widens the vortex core, the exchange energy decreases markedly. Thus, the gain in exchange energy when the vortex core reverses is very close to the sole exchange energy of the compressed core.

It is instructive to compare the profiles for a compressed vortex core with that corresponding to a Bloch point. Indeed, if only exchange energy were present, the system would feel no hindrance against introducing a BP as soon as the vortex exchange energy profile is above that of the BP for any radius. With standard micromagnetic programs, however, the computation of BP structure in equilibrium is only feasible in zero field, where one knows the BP location (see, however, Sec. III B). Therefore we compare the zero-field BP profiles discussed previously to those for the vortex. In the coarse mesh case first [Fig. 12(a)] the trend is clear: for a field just below the switching field the energy profiles are on the verge of lying at any radius $R$ above the BP profile. For the smaller mesh [Fig. 12(b)], the curves do not match so nicely, but the tendency remains.

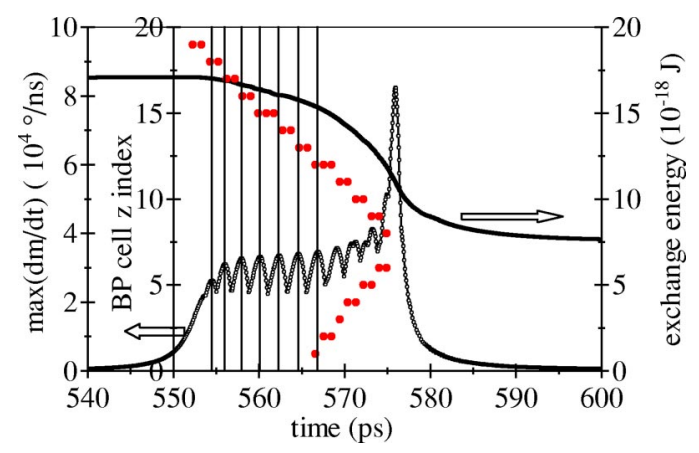

FIG. 10. Same calculation as for Fig. 8(b), but now the diameter is $200 \mathrm{~nm}$. Equilibrium and applied fields are 510 and $520 \mathrm{mT}$, respectively. Two Bloch points are injected, which annihilate together inside the film. The $z$ index of the cell that contains the BP is plotted, and vertical lines positioned at the torque maxima were drawn in order to correlate these with the BP cell-to-cell motion. 

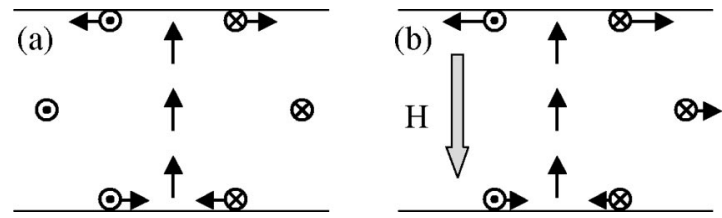

FIG. 11. Schematic drawing of the magnetization orientations in the vicinity of a vortex core. In equilibrium (a), surface charges appear due to the core magnetization, which induce a radial tilt of the moments at the surfaces, giving rise to spiraling magnetization flow lines (see Fig. 3). When a perpendicular field is applied suddenly (b), precession induces an asymmetric variation of these radial components: they increase at one surface, and diminish at the other.

One can try to improve this evaluation of exchange energy along a number of ideas, but this does not seem to be meaningful for the following three reasons: (1) we have neglected magnetostatic contributions in this analysis, and the total energy calculations show that they might be relevant at the scale of $10^{-19} \mathrm{~J} ;(2)$ at room temperature $25 k_{B} T$ is 1.0 $\times 10^{-19} \mathrm{~J}$, a barrier of this height will be overcome with an escape time of $1 \mathrm{~min}$ (for an inverse of the attempt frequency of $1 \mathrm{~ns}$ ); (3) $1.0 \times 10^{-19} \mathrm{~J}$ is the typical barrier height that remains in these profiles.

Varying the mesh and disk diameters, this analysis always gives the same type of results. Therefore, in spite of Aharoni's fears, the switching fields that were computed did not result from a numerical accident, but have a well-understood origin.

\section{B. Direct calculation of the energy barriers}

We now describe another approach that directly calculates the full micromagnetic energy barrier opposing vortex core reversal. A path method was recently developed ${ }^{24}$ to compute energy barriers that separate two micromagnetic states. In short, it considers a sequence of micromagnetic configurations that connect the two states, and relaxes all intermediate configurations until their local energy gradients are pointing along the path. The path finds saddle points as well as metastable states along its way. It was implemented on a finite elements micromagnetic code, so that it is possible to mesh finely where needed. This last feature is particularly important for the problem at hand, as the vortex core extends only over a small part of the disk. Several calculations were performed for a disk with $200 \mathrm{~nm}$ diameter, $50 \mathrm{~nm}$ thickness, and with a variation of the mesh size close to the disk center. In order to avoid the escape of the vortex outside of the finely meshed region, the following mesh was adopted (Fig. 13). The central zone, of diameter $20 \mathrm{~nm}$, was meshed with an average distance $d$ between nodes $(d=1,2,3$, or $4 \mathrm{~nm})$. In the middle region (radius between 10 and $20 \mathrm{~nm}$ ), the mesh size increased from $d$ to $4 \mathrm{~nm}$. In the outer ring (radius between 20 and $100 \mathrm{~nm}$ ), the mesh size increased from 4 to 10 $\mathrm{nm}$.

Figure 14 shows the computed paths (energy versus configuration number), at zero field and for different mesh sizes $d$ in the central zone. The number of configurations along the path was 25. The initial configurations (vortices with the same rotation sense, but with opposite core magnetizations) were equilibrated first. Then, the initial path was constructed by the linear rotation of all magnetization vectors. It means that the initial intermediate state is a $2 \mathrm{D}$ vortex (all vectors in the disk plane), with no core. The energy barrier for the initial path was very high, of the order of $6.2 \times 10^{-18} \mathrm{~J}$. The converged paths have much lower energy barriers (of the order of $2 \times 10^{-18} \mathrm{~J}$, see Table II). The saddle point (top of the energy along the path) is a configuration with a BP sitting in the middle of the film. The energy barriers (Table II) can thus be compared with the BP insertion energies computed in Sec. II (Table I). A very good agreement is found, despite the use of rather different programs and mesh types (and a magnetization $M_{s}=1 \mathrm{~T} / \mu_{0}=7.958 \times 10^{5} \quad$ instead of 8 $\left.\times 10^{5} \mathrm{~A} / \mathrm{m}\right)$.

Paths and barriers were then computed under an applied field. Zeeman energy increases the energy of the antiparallel state and decreases the energy of the parallel state. The energy barrier for the escape out of the metastable antiparallel state decreases with increasing field. In order to display this last feature better, energies have been plotted relatively to the energy of the antiparallel state in Fig. 15.
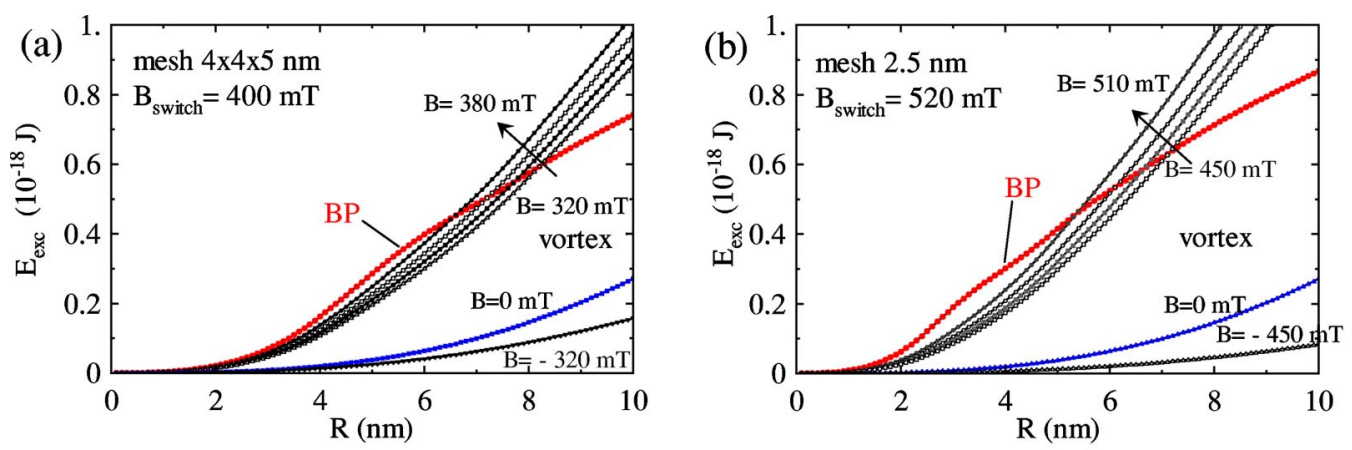

FIG. 12. Exchange energy within a half sphere of radius $R$, located on the sample top surface and centered on the vortex symmetry axis. The sample is $50 \mathrm{~nm}$ thick, of diameter $200 \mathrm{~nm}$, with a mesh size of $4 \times 4 \times 5 \mathrm{~nm}^{3}$ (a) and $2.5^{3} \mathrm{~nm}^{3}$ (b). The profiles are drawn at zero field and for several fields up to just below the computed core reversal field in each case (see Table I), as well as for one field parallel to the core magnetization. The exchange energy computed on the equilibrium structure of the BP, at the same mesh and zero field, is also plotted for comparison. 


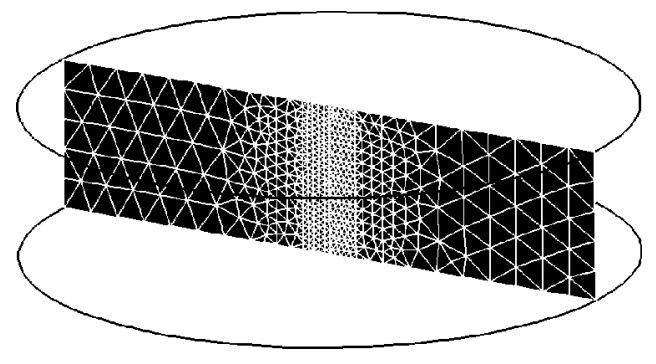

FIG. 13. Perspective view of the mesh used for the barrier calculations (200-nm-diameter and 50-nm-thick disk). The cut was made through one of the node planes used to generate the mesh. The finest mesh size is $2 \mathrm{~nm}$ in the central region.

The corresponding saddle-point configurations are shown in Fig. 16. They all display one Bloch point. Its vertical position shifts from the center of the film $(B=0)$ towards one film surface. The surface can be either the top or bottom one, depending on how the path calculation breaks the symmetry, and indeed both situations were obtained. The BP shift can be understood by looking again at Fig. 15, thinking that the horizontal axis now represents the vertical position of the BP. In a rough estimate the average magnetization in the field direction depends linearly on the BP position. The Zeeman energy adds a linear contribution to the total-energy profiles. As a consequence the position of the maximum shifts linearly with the strength of the applied field. When the barrier becomes close to zero, the saddle-point configuration has a BP that is very close to the surface. The field at which the energy barrier becomes zero is the switching field of the vortex core.

It is now instructive to plot the calculated barriers versus the applied field (Fig. 17). The variation with the field at low barrier is of the form $c\left(H_{s}-H\right)^{2}$, where $H_{s}$ has the meaning of an extrapolated field at zero barrier (switching field). There is no justification of this law at present, although similar forms are often used, sometimes with a different power. A much larger number of field points would be necessary to really test it. Refining the mesh leads to an increase of the barriers, and thus to an increase of the extrapolated switching

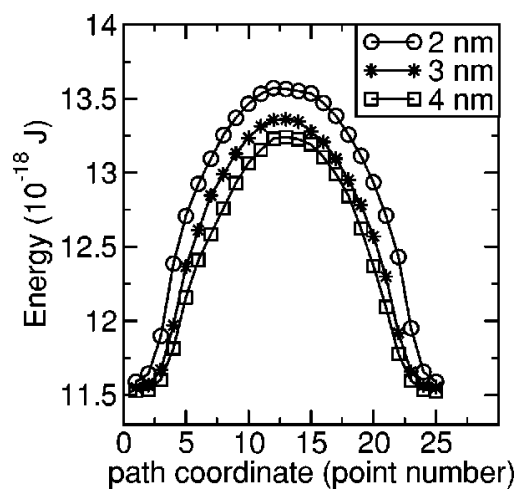

FIG. 14. Calculated minimum-energy paths for the reversal of the vortex core, at zero applied field. The use of a finer mesh in the central zone (the value of $d$ is indicated in the curves legend) leads to a higher barrier. Samples have a diameter of $200 \mathrm{~nm}$ and a thickness of $50 \mathrm{~nm}$.
TABLE II. Energies $\left(10^{-18} \mathrm{~J}\right)$ of the barrier state for a disk 200 $\mathrm{nm}$ in diameter, $50 \mathrm{~nm}$ thick, vs refined mesh size. The energies are counted in excess of that of a pure vortex.

\begin{tabular}{lrrrr}
\hline \hline Mesh $(\mathrm{nm})$ & 1 & \multicolumn{1}{c}{2} & \multicolumn{1}{c}{3} & \multicolumn{1}{c}{4} \\
\hline Total & 2.17 & 1.98 & 1.81 & 1.73 \\
Exchange & 2.26 & 2.13 & 1.98 & 1.90 \\
Demag. & -0.09 & -0.16 & -0.17 & -0.17 \\
\hline \hline
\end{tabular}

field $H_{s}$. As said before, switching can be considered to occur in a reasonable time as soon as the barrier is below $1 \times 10^{-19} \mathrm{~J}\left(\approx 25 k_{B} T_{a m b}\right)$. The switching fields determined by this calculation were also plotted in Fig. 7. They show a similar tendency to the points obtained by the quasistatic calculation of vortex core switching. The extrapolation of these data to zero mesh would not be very meaningful, considering their limited number.

\section{Extrapolation to zero mesh}

Now we try to extrapolate the results of the quasistatic calculations to zero mesh size. In principle, numerical micromagnetic calculations should be extrapolated to zero mesh size in order to get a proper mathematical solution of the continuous micromagnetic problem. The usual criterion is that the mesh size is smaller than the smallest characteristic length. For soft materials (low anisotropy) this is the exchange length $\Lambda=\sqrt{2 A / \mu_{0} M_{s}^{2}}$. This criterion is not sufficient here, as a singular structure is considered. In Sec. II, the extrapolation to zero mesh size of the BP exchange energy has been already considered, with the help of the analytic solution. There is no such analytic solution for a vortex under an applied field. Nevertheless, we calculate the exchange energy in half spheres of increasing radius $R$, centered at the sample top surface (Fig. 18). For the sake of comparing with Fig. 2, the parameters are the same (sample diameter and thickness, mesh sizes), and a fixed compressing field of 390 $\mathrm{mT}$ (applied $1^{\circ}$ off the normal) is applied. This field is just below the core switching field at the coarsest mesh. The two smaller meshes $(d=2.5$ and $3.125 \mathrm{~nm})$ give the same profile,

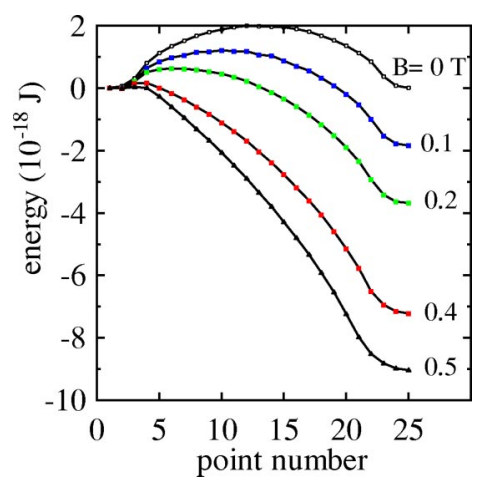

FIG. 15. Calculated minimum-energy paths for the reversal of the vortex core, for fields applied antiparallel to the core magnetization (induction values are indicated in $\mathrm{T}$ ). The energy reference is that of the metastable state. The mesh in the central region is $2 \mathrm{~nm}$. The sample dimensions are the same as in Fig. 14. 



FIG. 16. Saddle point states under an applied field (viewed in cut across the thickness), corresponding to Fig. 15. The images size is $100 \times 50 \mathrm{~nm}^{2}$, the field is oriented upwards and the color corresponds to the $z$ component of the magnetization unit vector [initial core magnetization is down (dark)]. The core mesh size is $2 \mathrm{~nm}$. Note the progressive magnetization rotation outside the core as the field increases, and the displacement of the BP saddle-point position towards the film surface.

while the larger mesh $4 \times 4 \times 5 \mathrm{~nm}^{3}$ is slightly above these profiles. The mesh dependence is much smaller than for the BP (compare with Fig. 2), and even of opposite trend. This is no surprise: a vortex, even compressed, is not a singular structure, contrary to the $\mathrm{BP}$, so that as soon as the mesh size is below the exchange length, the computed structures should not change. As a consequence, we will neglect the change with mesh of the vortex exchange energy in our analysis.

The mathematical result of this extrapolation is clear. An infinite field is necessary to inject a BP at zero mesh size, because a $R^{2}$ profile (the rate of increase of the exchange energy in the vortex core, see Fig. 12) can never lie above a linear $(R)$ one (the rate for the $\mathrm{BP}$ ) for all $R$. Physically, the region where the BP profile is above the compressed vortex one will shrink with increasing field, and the maximum difference between the two (equivalent to a barrier) will decrease. Taking into account thermal activation, a switching

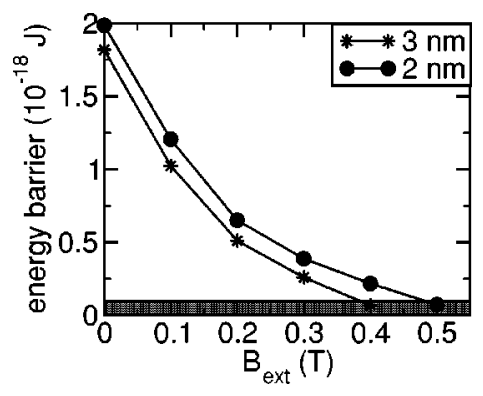

FIG. 17. Plot of the energy barrier height vs the applied field, for two different core mesh sizes. The barrier decreases under the applied field roughly as a parabola, and a parabolic extrapolation from the four high-field points gives switching fields $\mu_{0} H_{s}=490 \mathrm{mT}(3$ $\mathrm{nm})$ and $660 \mathrm{mT}(2 \mathrm{~nm})$. The shaded region corresponds to a barrier height below a thermal threshold $\left(25 k_{B} T\right.$ at room temperature). Mesh refinement increases the calculated barriers, as seen before.

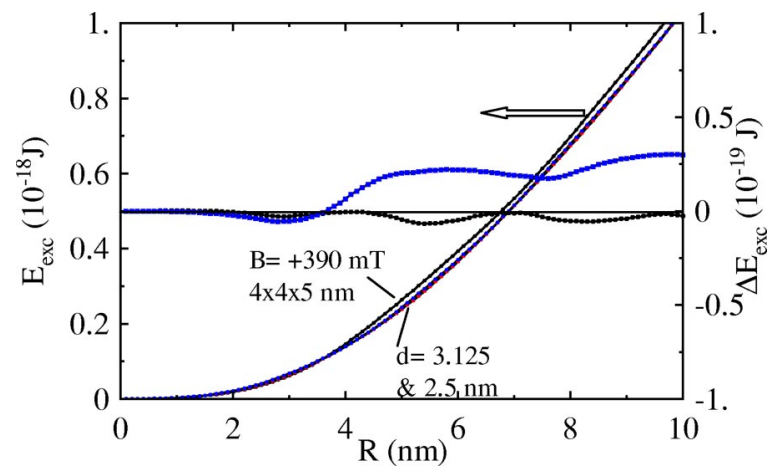

FIG. 18. Plot of the local exchange energy in a half sphere centered on the vortex core center and located at the film surface, for decreasing mesh size. Contrarily to the BP structure (Fig. 2), the change with the mesh size is very small. The two smaller meshes give here the same results. The right axis magnifies the energy differences with respect to the profile at $d=2.5 \mathrm{~nm}$; only for the larger mesh can a small change be seen. The sample is $50 \mathrm{~nm}$ thick with a diameter of $200 \mathrm{~nm}$, and the field (at $1^{\circ}$ ) is $390 \mathrm{mT}$, just below the core switching field for the larger mesh.

field can be defined as the point where the barrier height becomes smaller than $1 \times 10^{-19} \mathrm{~J}$. We estimate it by extrapolation (with a second-order polynomial) of the profiles computed at fields strengths below the switching. This results in fields of the order of $500 \mathrm{mT}$ at a diameter of $100 \mathrm{~nm}$, and $700 \mathrm{mT}$ at a diameter of $200 \mathrm{~nm}$. They lie above the fields computed at the smallest mesh, namely, 350 and $510 \mathrm{mT}$, respectively. These values are also above the experimental ones. However one should not forget that these extrapolations are based only on the exchange energy, neglecting the magnetostatic contribution, and that they may depend strongly on the way the exchange energy is evaluated, both at mesh points and within the considered spheres. On the other hand, experimental values seem to depend on the sample quality. For a $400-\mathrm{nm}$ disk with $50 \mathrm{~nm}$ thickness, mean core switching fields of 380 and $450 \mathrm{mT}$ were found for different samples (maximum switching fields being 500 and $600 \mathrm{mT}$, respectively). ${ }^{17}$ A likely possibility is that defects may favor the nucleation of the BP, thus lowering the switching field. In this respect, we note that experiments by another group using $1-\mu \mathrm{m}$-diameter and 80 -nm-thick Permalloy disks have found by repetitive experiments that the switching field for a single disk shows a distribution, similar to that of an array of disks. ${ }^{25}$ Other recent experiments by Okuno ${ }^{17}$ have also shown that every disk has a switching field distribution of finite width, albeit smaller than the distribution measured on an array of disks. This indicates a non-fully-deterministic process, consistent with the role of both defects and thermal agitation.

Empirically, we found that the calculations we performed at $d=3.125 \mathrm{~nm}$ are close to the mean switching fields both at a thickness of $50 \mathrm{~nm},{ }^{16}$ and when decreasing thickness down to $15 \mathrm{~nm}$, where a strong reduction of the core switching field was measured. ${ }^{17}$ In contrast to this finding, extrapolated fields prove rather insensitive to sample thickness; they even increase because of the shape effect (thinner samples are harder to magnetize perpendicularly). This arises from the 
independence of the BP exchange energy profile at small radius from the sample macroscopic dimensions, in agreement with the anaytical profile of the core vicinity. One could interpret these results by considering that a finite mesh mimics sample imperfections but, clearly, this idea awaits justification.

\section{CONCLUSION}

In conclusion, this work has shown first that standard micromagnetic calculations that allow the presence of a Bloch point are possible. The Bloch point is of course always located between mesh points. Exchange energy profiles extracted from the micromagnetic calculations were found to agree with the analytic profile, with a difference appearing at distances below the mesh size. This gives an underestimated BP energy, which however should play no role in calculations that only consider the displacement of an existing BP. A mesh-friction effect has also been detected in the calculations, which partly impedes the BP motion. The micromagnetic extension of the BP structure has been determined by these calculations, and a radius of $10 \mathrm{~nm}$ was found.

The calculations under field have also shown that, as expected from topological reasons, the vortex core reversal under an antiparallel field involves the nucleation of the BP at one sample surface, followed by its displacement across the sample thickness. This problem of inserting a BP into a sample, from outside, appears to be more complex because here the BP energy plays a role. We have studied it by two types of micromagnetic calculations, either by a dynamic calculation (high or normal damping) with a step-by-step increase of the applied field, or by the evaluation of the minimum-energy path in the full micromagnetic configuration space. The calculated switching fields show a strong mesh dependence, but nevertheless are of the order of magnitude of the experimental values at reasonable mesh sizes $(d \leqslant \Lambda)$. We have developed a local analysis, focused on the exchange energy contribution, which predicts that infinite fields would be needed at zero temperature and zero mesh size, and of course in a defectless sample. Extrapolation to zero mesh but at room temperature predicts fields that are higher than the experimental values obtained up to now, so that the BP insertion in the experiments is probably assisted by defects.

\section{ACKNOWLEDGMENTS}

This work was supported by a NEDO international joint research grant Nanopatterned magnetic systems: magnetic structures and dynamical behaviors. The research of J.M.G. was supported by the EC. The stay at Orsay of R.D. was supported by the EC (site ETNANOMAG, Contract No. HPMT-CT-2000-00066). We thank T. Okuno, T. Shinjo, and Y. Suzuki for helpful discussions, and T. Okuno for the communication of experimental results before publication.

\section{APPENDIX: DETECTION AND LOCALIZATION OF A BLOCH POINT IN A MICROMAGNETIC CONFIGURATION}

We use the defining property that the magnetization vectors of the points on a spherelike surface that surrounds a BP

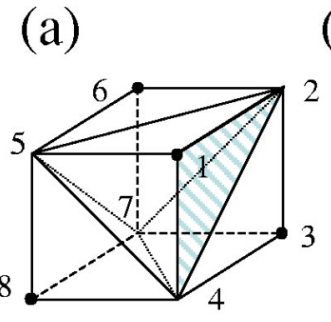

(b)

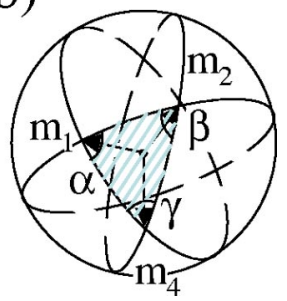

FIG. 19. Eight neighboring mesh points (a) and the image on the unit sphere of the triangle whose corners are the points 1,2 , and 4 (b).

must cover exactly the unit sphere. Here, for a cubic mesh the natural spherelike surface is a cube built from eight cells. The apparent difficulty is that the computed structure is by necessity discrete, so that one does not know the orientation of the magnetization vector at any place on this surface. Nevertheless, as the magnetization is assumed to be continuous (except at BP's, but they are in the interior of the cubes in most cases), we can estimate this surface as follows.

Consider a cube whose eight corners are nearest-neighbor mesh points. To each triangle drawn on the cube surface corresponds a triangle on the unit sphere (Fig. 19). The corners of this second triangle are the magnetization directions at the three points considered on the cube. If we knew the full magnetization distribution, this triangle would be mapped on the unit sphere as a spherical triangle with distribution dependent edges shapes. Not knowing the distribution of $\vec{m}$, convenient triangles on the sphere are those whose edges are arcs of great circles [Fig. 19(b)]. For then, by Gauss formula, the area $T$ of such a triangle is

$$
T=\alpha+\beta+\gamma-\pi,
$$

where $\alpha, \beta$, and $\gamma$ are the angles within the triangle. They are easily computed from the magnetization vectors as

$$
\cos \alpha=\frac{\left(\vec{m}_{1} \times \vec{m}_{2}\right) \cdot\left(\vec{m}_{1} \times \vec{m}_{4}\right)}{\left|\vec{m}_{1} \times \vec{m}_{2}\right|\left|\vec{m}_{1} \times \vec{m}_{4}\right|}, \quad \text { etc. }
$$

Of course Eq. (A1) does not evaluate the exact area of the triangle with nonregular edges corresponding to the three points considered plus all the intermediate ones. But, as we are only interested in the total surface, all the errors will add to zero when we sum over the 12 similar triangles on the cube.

This is the first method developed. In most cases it works, and one obtains either $S=\Sigma_{p} T_{p}=4 \pi$ to double precision, or less than $2 \pi$. But it may fail when the BP is very close to one facet. It has also the drawback that all surfaces are counted as positive, whereas one should get either 0 (no BP), or $\pm 4 \pi$ (one BP), or $\pm 8 \pi$ (two BP's of the same sign), etc. For this, the orientation of the surfaces should be taken into account.

In order to obtain a more precise BP position, we group the triangles according to a corner that they share [this sorts out four of the eight corners, see Fig. 19(a)]. The partial sums thus formed $S_{1}, S_{3}, S_{6}$, and $S_{8}$ are equal (to $\pi$ ) if the 


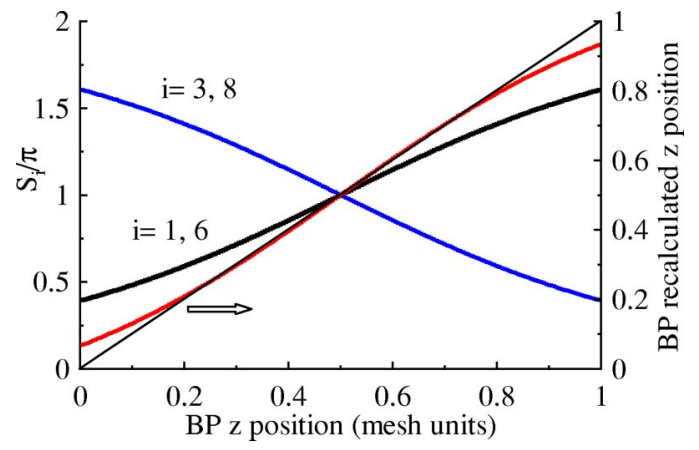

FIG. 20. Variation of the four partial area sums with $z$ position of a BP inside a cube. The calculation was performed using the analytic hedgehog configuration. In the central region, the sums depend linearly on the BP position. The recalculated BP position according to Eq. (A3) is also plotted.

$\mathrm{BP}$ is exactly in the center of the cube (think of the analytic configurations in Fig. 1), and increase if the BP is closer to the respective corner. Figure 20 shows how a vertical motion of the BP affects the sums.

If we take for simplicity the tangent to these curves at the center, we get the following approximate refined BP position, with respect to the cube center (cube size $d$ ):

$$
Z \approx \frac{0.1786 d}{\pi}\left(S_{1}+S_{6}-S_{3}-S_{8}\right)
$$

The linearization is justified as the BP prefers to sit between the mesh points, as seen before.

The second method developed is more robust. It considers for every corner the magnetizations of its nearest neighbors (see Fig. 21, drawn for corner 1 in Fig. 19). If the magneti-
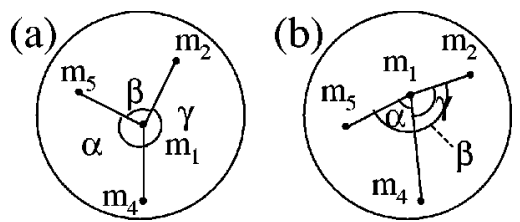

FIG. 21. The three magnetizations of the three neighbors of one point can either surround its magnetization (a) or not (b). In a Bloch-point structure, case (a) has to occur for every corner. The occurrence of this case is detected by the sum of the three angles on the sphere.

zation distribution covers the sphere, the magnetization vectors in surrounding (on the spherelike surface) of a point also have to surround the magnetization of this point on the unit sphere. For example, the three nearest neighbors have to display this property. In that case the sum of the three angles formed amounts to $2 \pi$ [Fig. 21(a)]. In the opposite case the sum is smaller [Fig. 21(b)]. Therefore, a cube where for all corners the sum of the three angles is $2 \pi$ contains one BP. Indeed, the covered surface on the sphere will be, from Eq. (A1),

$S=8($ No. of corners $) \times 2 \pi-12$ (No. of triangles $) \times \pi=4 \pi$.

This second procedure is more handy because it splits the check of BP presence into eight partial checks that all must pass. But it does not help in localizing the BP within the cube. It also does not take care of the orientation, so that a configuration without a BP does not yet result in a zero total surface. In practice, a tolerance criterion has to be used ( $S$ $>3.8 \pi$, for example) in order to check that no BP's are missed.
*Email address: thiaville@1ps.u-psud.fr

${ }^{1}$ E. Feldtkeller, Z. Angew. Phys. 19, 530 (1965).

${ }^{2}$ M. Kléman, Points, Lines and Walls (Wiley, Chichester, 1983).

${ }^{3}$ P.R. Kotiuga, IEEE Trans. Magn. 25, 3476 (1989).

${ }^{4}$ C. Harrison and K. Leaver, Phys. Status Solidi A 15, 415 (1973).

${ }^{5}$ T. Beaulieu, B. Brown, B. Calhoun, T. Hsu, and A. Malozemoff, in Magnetism and Magnetic Materials-1976, edited by J. J. Becker and G. H. Lander, AIP Conf. Proc. 34 (AIP, New York, 1976), p. 138.

${ }^{6}$ Y. Kabanov, L. Dedukh, and V. Nikitenko, Pis'ma Zh. Eksp. Teor. Fiz. 49, 551 (1989) [JETP Lett. 49, 637 (1989)].

${ }^{7}$ A. Thiaville and J. Miltat, Europhys. Lett. 26, 1006 (1994).

${ }^{8}$ W. Brown, Jr., Micromagnetics (Interscience, New York, 1963).

${ }^{9}$ W. Döring, J. Appl. Phys. 39, 57 (1968).

${ }^{10} \mathrm{~J}$. Slonczewski, in Magnetism and Magnetic Materials-1975, edited by J. J. Becker, G. H. Lander, and J. J. Rhyne, AIP Conf. Proc. 29 (AIP, New York, 1976), p. 613.

${ }^{11}$ M. Margulies and J. Slonczewski, J. Appl. Phys. 49, 1912 (1978).

${ }^{12}$ A. Hubert, J. Magn. Magn. Mater. 2, 25 (1976).

${ }^{13}$ J. Reinhardt, Int. J. Magn. 5, 263 (1973).

${ }^{14}$ Y. Nakatani and N. Hayashi, IEEE Trans. Magn. 24, 3039 (1988).
${ }^{15}$ Y. Nakatani and N. Hayashi, IEEE Trans. Magn. 29, 2587 (1993).

${ }^{16}$ T. Okuno, K. Shigeto, T. Ono, K. Mibu, and T. Shinjo, J. Magn. Magn. Mater. 240, 1 (2002).

${ }^{17} \mathrm{~T}$. Okuno (private communication).

${ }^{18}$ oOMmF was developed by M. J. Donahue and D. Porter mainly, from NIST. It is available at http://math.nist.gov/oommf; we used the $3 \mathrm{D}$ version of the $1.2 \alpha 2$ release.

${ }^{19}$ A. Aharoni, J. Appl. Phys. 51, 3330 (1980).

${ }^{20}$ A. Hubert and R. Schäfer, Magnetic Domains (Springer-Verlag, Berlin, 1998).

${ }^{21}$ There is even a toy built on this principle, see http:// www.geomag.it

${ }^{22}$ P. Trouilloud and J. Miltat, J. Magn. Magn. Mater. 66, 99 (1987).

${ }^{23}$ A. Aharoni, in Magnetic Hysteresis in Novel Magnetic Materials, Vol. E338 of NATO Advanced Studies Institute, Series E: Applied Science, edited by G. Hadjipanayis (Kluwer Press, Dordrecht, 1997), pp. 1-20.

${ }^{24}$ R. Dittrich, T. Schrefl, D. Süss, W. Scholz, H. Forster, and J. Fidler, J. Magn. Magn. Mater. 250, 12 (2002).

${ }^{25}$ N. Kikuchi, S. Okamoto, O. Kitakami, Y. Shimada, S. Kim, Y. Otani, and K. Fukamichi, J. Appl. Phys. 90, 6548 (2001). 